\title{
EL APRENDIZAJE DEL ESTUDIANTE UNIVERSITARIO COMO SISTEMA VIVO
}

\section{THE LEARNING OF THE UNIVERSITY STUDENT AS A LIVING SYSTEM}

\section{Referencia del artículo}

Zapet, A. (2020). El aprendizaje del estudiante universitario como sistema vivo. Revista Científica del SEP. 3(I), 67-7I.

DOI: https://doi.org/10.36958/sep.v3i01.38

\author{
Aura Patricia Zapet Serrano \\ patyzapetser@yahoo.es \\ Licenciada en Pedagogía y Ciencias de la Educación \\ Centro Universitario de Occidente -CUNOC \\ Universidad de SanCarlos de Guatemala \\ Fecha de recibido:25/06/2020 \\ Fecha de aceptado: $30 / 10 / 2020$
}

\section{RESUMEN}

El sistema vivo que representa el estudiante universitario, está organizado por la sinergia de la razón y la emoción, que representa la conciliación entre la manifestación de las emociones y la construcción cognitiva, como principios fundamentales para la vida del ser humano.

Todas las emociones son parte de la complejidad de cada ser, permiten reaccionar ante diferentes circunstancias y brindan un estado homeostático al cuerpo y la mente para su evolución en el mundo moderno, esto explica la naturaleza esencial del ser humano como sistema vivo, sus impulsos, reacciones y la toma de decisiones.

Cuando amplí-a su visión, puede tomar conciencia del placer y la tensión necesarios para el aprendizaje, como parte de la diversidad de experiencias que cumplen una función importante al identificar el umbral necesario para su beneficio y rechazar los altos niveles que afecten el funcionamiento equilibrado de su sistema.

El estudiante universitario necesita comprender la biologí-a de su aprendizaje, identificar que la convivencia, experiencias e influencias del entorno impactan la información de su estructura celular, como aporte epigenético para los cambios, en donde sus creencias constituyen el pilar fundamental.

\section{PALABRAS CLAVE}

Sistema vivo, biología y aprendizaje

\section{ABSTRACT}

The living system represented by the university student is organized by the synergy of reason and emotion, which represents the conciliation between the manifestation of emotions and cognitive construction, as fundamental principles for the life of the human being.

All emotions are part of the complexity of each being, they allow to react to different circumstances and provide a homeostatic state to the body and mind for its evolution in the modern world, this explains the essential nature of the human being as a living system, its impulses, reactions and decision making.

When you broaden your vision, you can become aware of the pleasure and tension necessary for learning, as part of the diversity of experiences that play an important role in identifying the necessary threshold for your benefit and rejecting the high levels that affect the balanced functioning of your system.

The university student needs to understand the biology of their learning, identify that coexistence, experiences and environmental influences impact the information of their cellular structure, as an epigenetic contribution to changes, where their beliefs constitute the fundamental pillar.

\section{KEYWORDS}

Living system, biology and learning 


\section{I.- INTRODUCCIÓN}

Comprender como aprende el estudiante universitario desde su biología, conlleva un acercamiento a las concepciones que explican las emociones y los procesos racionales, como dos estructuras separadas pero complementarias que enriquecen las experiencias de aprendizaje, en donde el estudiante siente, piensa, descubre, resuelve problemas y enfrenta nuevos desafíos.

El reconocimiento de las emociones, apertura la posibilidad de experiencias placenteras en el entorno de aprendizaje, el placer es un elemento necesario que se percibe como la energía capaz de producir efectos en la sensibilidad y pensamiento del estudiante y lo predispone para aprender, por otra parte, la tensión que es interpretada en el cuerpo como el estrés, es parte de la diversidad de experiencias químicas del cuerpo que contribuyen a su desarrollo, desde la gestión equilibrada.

La construcción del aprendizaje se da a lo largo de la vida, colmado de vivencias y creencias de cada ser humano, es parte de la historia, herencia, lenguaje, cultura, religión e influencias sociales, pero en la educación universitaria, la mediación del docente es fundamental, para orientar la reestructuración de procesos integrales que abren las posibilidades al desarrollo de los estudiantes.

\section{2.- MÉTODOS}

La investigación realizada responde al enfoque cualitativo, en búsqueda de un acercamiento más profundo a la intervención de la cognición y la emoción en el aprendizaje del estudiante universitario de la Carrera de Trabajo Social del Cunoc, para ello se contó con la participación de estudiantes y docentes como protagonistas del entorno, lo que permitió tomar en cuenta diferentes puntos de vista y experiencias, de mucha utilidad para interpretar y reconstruir el contexto desde un proceso inductivo.

Con apoyo en los métodos, hermenéutico; que facilita el análisis e interpretación de diferentes aportes teóricos y fenomenológico; que orienta la interpretación de la experiencia vivida desde la perspectiva del participante, permitió comprender el sentido de las diferentes expresiones manifestadas en el diálogo con los informantes. Para ello se utilizaron entrevistas semi estructuradas con estudiantes cursantes del octavo y décimo semestre de la carrera de Trabajo Social del Cunoc y se recurrió a sostener conversaciones con los docentes que impartieron clases en estos semestres.

El objetivo general de la investigación se basó en analizar el vínculo entre cognición y emoción para el desarrollo del aprendizaje del estudiante universitario, detectar si los docentes toman en cuenta dicho vínculo en su docencia y a su vez, si los estudiantes tienen conocimiento de sus propias emociones y su implicación al aprender, lo cual demandó la interpretación de diferentes aportes teóricos sobre las emociones y los procesos racionales, como la Teoría de la Cognición de Varela y Maturana, el Senti Pensar desde la perspectiva de Galeano, la disposición al cambio a través de la Biología Celular que explica Bruce Lipton, o el aporte de las Neurociencias para el Aprendizaje del estudiante y la Formación del docente.

\section{3.- RESULTADOS, ANÁLISIS Y DISCUSIÓN}

\section{EL APRENDIZAJE DEL ESTUDIANTE UNIVERSITARIO COMO SISTEMA VIVO}

La interpretación del ser humano como sistema vivo, tiene diferentes acepciones, desde la época antigua se cree en el dualismo del ser humano conformado por dos estructuras: el alma y el cuerpo; mientras el alma es espiritual, inmortal y pertenece al mundo de las ideas, el cuerpo representa la parte sensible y material, un recipiente para el alma. Desde esta postura las ideas no son producto de sensaciones sino del pensamiento y se crea una separación de estructuras con trascendencia en la educación.

Los filósofos como Sócrates, Platón y Aristóteles, creyeron en la primacía del alma, derivado de una visión espiritual del universo que les permitió construir afirmaciones como "Las cosas del alma o mente están antes que las cosas del cuerpo”, esto admite comprender la importancia de la mente sobre el cuerpo, como sustancia superior o como esencia de la vida, de esta manera se concibe que la mente y el cuerpo poseen sus propias cualidades, pero el pensamiento es superior a los sentidos, porque los sentidos son funciones del cuerpo.

Desde otra postura, la filosofía occidental con el aporte de Descartes a través del método racional, permite interpretar la valoración del razonamiento como punto de partida, creer en la razón como el nivel más desarrollado a partir del cual se manifiestan premisas para explicar el desarrollo del ser humano, lo cual vino a fortalecer la concepción dualista alma y cuerpo, pero en la dinámica del ser humano prevalece el reconocimiento a la razón.

La creencia en la razón como área más desarrollada se atribuye a funciones del cerebro, sin embargo, el instinto mismo de supervivencia se debe a las emociones, en donde intervienen estructuras como la amígdala cerebral que permite una reacción inmediata de protección, se manifiesta con más rapidez, porque el área del cerebro responsable de las reacciones emocionales es más evolucionada y rápida ante el tipo de experiencias impredecibles, pero en seguida es complementada con el razonamiento. 
El ser humano representa la unidad como "un sistema organizado" permite conocer la complejidad de sus funciones integradas por millones de células que conforman su estructura orgánica, para llevar a cabo las funciones necesarias como un sistema abierto que intercambia materia y energía con su entorno natural y contexto social, el reconocimiento de su estado indivisible permite comprender la relación complementaria entre la razón y las emociones para su disposición al aprendizaje.

Uno de los hallazgos en la investigación, es la tendencia a ignorar la participación de las emociones en el proceso de construcción del aprendizaje, dichos procesos son orientados a la construcción cognitiva, cantidad y calidad de información. La indiferencia o la represión de las emociones, son indicadores de alerta, el estudiante necesita aprender a identificar su presencia y autorregular su efecto para aprovechar la energía de cada emoción y disposición biológica para el aprendizaje, es parte de lo que se conoce como educar las emociones.

Las emociones trascienden la vida del estudiante, provocan una serie de cambios porque el sistema nervioso y todo el cuerpo se encuentran implicados, estos cambios pueden ser observables o no, quizá lo más importante de las emociones es que provocan en el interior del cuerpo la segregación hormonal - liberación de neurotransmisores que en consecuencia, disponen al organismo a procesos más elaborados que son parte de los procesos cognitivos, para prestar atención, analizar, sintetizar, comparar, reflexionar y dar respuesta al estímulo recibido.

Las emociones son parte de la riqueza de cada ser humano porque le permiten reaccionar ante diferentes circunstancias $y$ brindan un estado homeostático al cuerpo y la mente para su evolución en el mundo moderno, no es posible vivir en una sola tendencia de emociones, todas son necesarias porque diversas son las situaciones que se viven cotidianamente, el problema detectado en la investigación que se llevó a cabo es que el estudiante no relaciona la vivencia de sus emociones con su proceso de aprendizaje, solamente como parte de la vida en general.

La desconexión que el estudiante tiene de las emociones con su aprendizaje, limita reconocer el efecto placentero, Hugo Assmann, admite que "el conocimiento emerge en su dimensión vitalizadora cuando tiene algún tipo de vinculación con el placer" por lo tanto, esta experiencia debe ser percibida durante el aprendizaje. Desde esta perspectiva, el placer es como el adhesivo que funde el vínculo de la emoción con los procesos cognitivos para producir el aprendizaje y el estudiante debe tener conciencia de ello.

De manera natural, el ser humano brinda respuesta a las provocaciones agradables, fisiológicamente esta reacción desencadena agentes químicos que el mismo cuerpo produce como la dopamina, que predispone al aprendizaje, porque interviene en el sistema para experimentar bienestar $y$ aumenta la capacidad de memoria, atención y concentración, como funciones cognitivas fundamentales para el aprendizaje, los recuerdos se consolidan o están vinculados a por lo menos una emoción, de ahí la importancia de las emociones positivas que propician bienestar en la experiencia del estudiante universitario.

Por otra parte, la vida misma demuestra que no todas las experiencias o emociones son agradables, también existe la tensión para el aprendizaje, provocada por la adrenalina o cortisol, pero son parte del mismo sistema, permiten reaccionar, buscar nuevas soluciones y enfrentar las circunstancias o nuevos retos que aperturan otras experiencias de aprendizaje, en su conjunto todas las emociones contribuyen al equilibrio sistémico del ser humano, todo depende de la percepción y gestión de las mismas, porque también se conoce que el exceso de estrés puede enfermar al cuerpo y la mente, lo que deriva un comportamiento poco favorecedor para su desarrollo.

Desde el contexto universitario, el estudiante se enfrenta a diferentes estresores, que son parte de su vida y que debe aprender a manejar para su formación y preparación profesional, de ahí la importancia de aprender a interpretar el estrés necesario para su beneficio y rechazar los altos niveles que afecten el funcionamiento equilibrado de su organismo, como lo expresa Luis Gaviria en su libro "Aprovechando el Estrés para Triunfar".

La tensión que experimenta el estudiante universitario al enfrentarse al campo de estudio de su carrera, o malas relaciones educacionales, podrían afectar su desarrollo, si la percepción construida tiene esa inclinación, pero la tensión como desafío fortalece su sistema porque aprovecha la energía que la presión o estrés le provoca para gestionar sus emociones y continuar su desarrollo.

Las emociones prevalecen en la convivencia entre estudiantes y docentes, están presentes en las palabras, acciones e intenciones, que tienen una frecuencia vibratoria que afecta sus relaciones de manera positiva o negativa, lo que provoca que el estudiante en el aula preste más atención a esa energía que al curso que recibe.

En la investigación, se detectó que la transferencia del docente se lleva a cabo por medio del lenguaje, ya sea gestual, mímico o verbal y la mediación pedagógica que implementa impacta en las personas que se encuentran a su alrededor, si la energía es negativa perjudica al estudiante produciendo inseguridad e intimidación y ante la amenaza interpretada el estudiante se cohíbe y no participa. 
Por otra parte, el estudiante universitario se enfrenta al desconocimiento de la construcción de su propio aprendizaje, desconoce la intervención de las emociones en la biología de su aprendizaje, tampoco tiene conciencia del efecto de sus buenas o malas relaciones personales para su disposición como sistema aprendiente.

Es necesario que el estudiante universitario tome conciencia de sus emociones, para autorregular sus efectos en el cuerpo y pensamiento, comprender que los estímulos percibidos le producen cambios en toda su estructura, y de esta interrelación se predispone a brindar respuestas.

Desde los aportes de Galeano, "Si las emociones y sentimientos provocan cambios estructurales químicos, energéticos y neurales, podemos entonces deducir que el fluir del sentipensar es también un fenómeno biológico que envuelve cambios estructurales de la organización viva." (Galeano, 20I2)

En estos términos, el proceso educativo no se puede dirigir a la estructura cognitiva sino a la estructura viva, este es el significado de completud del ser, senti pensar se convierte en el lenguaje emergente para desarrollar el sistema interno en correspondencia a sus relaciones externas.

\section{4.- EL APRENDIZAJE DEL ESTUDIANTE UNIVERSITARIO}

La actividad genera experiencia como medio de interacción que permite un acercamiento con el entorno, obtener información, organizarla y formar esquemas de representación a través del pensamiento como parte del aprendizaje racional, así los procesos del pensamiento que intervienen propician la construcción desde la individualidad de cada ser aprendiente, en donde se relacionan armónicamente la emoción y la razón.

En la construcción del aprendizaje no se pueden separar las emociones de los procesos cognitivos, Maturana plantea que, "los actos humanos se fundamentan en los argumentos que construimos al concatenar las acciones que son significativas para nosotros" para ello debe existir intensidad en el estado emocional, esa energía que orienta los razonamientos acorde a la experiencia vivida, que insta la toma de decisiones y desde la huella de la emoción construye registros para nuevas experiencias conscientes e inconscientes.

El aprendizaje surge de la relación de la experiencia y las creencias de cada ser humano, como germen para el conocimiento, en él interviene la estructura cognoscitiva, que representa los procesos más elaborados del sistema de cada ser, la otra parte está conformada por las emociones que representan el mundo desconocido y un nuevo reto para promover los procesos de aprendizaje.
La teoría de Santiago de la cognición, propuesta por Humberto Maturana y Francisco Varela, explica el proceso de conocimiento a lo largo de la vida, la cognición desde esta teoría no es una actividad que corresponda al cerebro, sino al ser humano desde todos sus ámbitos: biológico, social, espiritual, cultural o histórico, una interrelación que provoca la disposición para el aprendizaje. Esto nos permite comprender que todo cambio en la convivencia, las relaciones y la experiencia, contribuye a la modificación biológica del ser, desde la transformación del ADN de la célula, dichos cambios producen pensamientos y comportamientos diferentes que se explican desde el aporte epigenético, de ahí la importancia de plantear la educación como una diversidad de experiencias.

La disposición al cambio es propia de los seres humanos, una condición que permite explicar el aprendizaje, se promueve con la variación en los estilos de vida, cambios del entorno, alimentación, actividad física y mental, entre otros, lo importante es la incidencia en el ADN para asegurar la permanencia en los registros de memoria celular. Al respecto, Rocío Corral aporta lo siguiente, "si nos mantenemos firmes con una emoción, actitud o tipo de pensamiento, durante largo tiempo, lo que estamos haciendo es reforzar los receptores de esas células hacia la recepción de un tipo de química" (Corral, 20l4).

En otras palabras, el estudiante universitario que cambia sus hábitos de estudio, su comportamiento y estilo de vida, lo alcanza desde la biología de su sistema y recurrencia de sus acciones, formando registros de memoria en el sistema nervioso, cerebro y todo el cuerpo.

El estudiante universitario necesita conocer la biología de su aprendizaje, identificar el ambiente y las influencias del entorno que afectan su estructura celular e impactan la información de su sistema interno, Bruce Lipton explica que "no estamos determinados por nuestros genes, sino condicionados por el entorno y sobre todo por nuestras creencias, somos dueños absolutos de nuestro destino" (Lipton, 2015).

Esta abstracción, puede inspirar al estudiante a tomar responsabilidad de las condiciones que le permiten aprender, conocer su interior, reconocer el poder de sus pensamientos, sus acciones e interpretar las influencias de su entorno.

Uno de los mayores retos de la educación universitaria es la transformación del ser humano, Romero Lárez, en su libro Educar para la vida en la universidad contemporánea, afirma que "es necesario dar paso de la universidad informadora a la universidad creadora", la trasformación de cada ser humano involucra sentir, pensar y actuar, sustentada en que cada célula tiene su propia organización y reacción química, 
produceenergía, elimina la sustancia innecesaria, da respuesta a los estímulos del ambiente e intensifica su actividad a través de la comunicación con otras estructuras, esto lo explica Bruce Lipton en su libro la Biología de la Transformación y se refiere al poder e influencia del ser humano en su propia evolución.

Para dar paso a la transformación, es necesario dudar de la certeza, reconocer la importancia de los cambios que el estudiante puede alcanzar desde su estructura biológica y los beneficios que la docencia le pueda brindar, las neurociencias refieren que "Enseñar sin conocer el cerebro es como hacer un guante sin conocer la mano", así docente y estudiante no podrán cambiar sin comprender como se lleva a cabo este proceso y cómo se disponen para el aprendizaje.

\section{5.- CONCLUSIONES}

Para educar no basta con la experiencia y conocimiento científico, es importante que el docente conozca la construcción biológica del aprendizaje, para permitir que el estudiante aprenda desde la integración de sus emociones en complementariedad con los procesos cognoscitivos.

Para que estudiantes y docentes cambien su interpretación de aprendizaje, es necesario que la educación universitaria trascienda de informadora a creadora, libre de prácticas amenazantes y condicionamientos que saturan de prácticas observables, cuantificables y medibles que limitan el desarrollo de la integralidad del estudiante como sistema vivo.

\section{6.- REFERENCIAS BIBLIOGRÁFICAS}

Corral, R. (8 de agosto de 20I4). CAMBIA EL PENSAMIENTO Y MODIFICA TUS GENES!!!! Obtenido de https://conexiondelfin. blogspot.com/2014/08/cambia-el-pensamiento-y-modificatus_8.html

Galeano, E. (2 de mayo de 20I2). SENTI - PENSAR . Obtenido de https://senti-pensar.blogspot.com/20 I 2/05/el-libro-de-losabrazos-egaleano.html

Lipton, B. H. (25 de abril de 20 I 5). Frecuencia Gaia. Obtenido de La Biología de la Creencia: https://frecuenciagaia.com/2015/04/25/ bruce-h-lipton-la-biologia-de-la-creencia/

Salazar, S. F. (2005). EL APORTE DE LA NEUROCIENCIA PARA LA FORMACIÓN DOCENTE. "Actualidades Investigativas en Educación", 10.

\section{Sobre autora}

\section{Aura Patricia Zapet Serrano}

Es Docente Interina del Centro Universitario de Occidente CUNOC, de la Universidad de San Carlos de Guatemala, Directora Técnica Administrativa de Haskala Centro Educativo Integral, más de 20 años de experiencia en los diferentes niveles educativos del Sistema de Educación de Guatemala. Licenciada en Pedagogía y Ciencias de la Educación y Msc. En Docencia Universitaria, egresada del CUNOC de la Universidad de San Carlos de Guatemala.

La educación necesita ser inspiradora para dar oportunidad a la creación, desarrollar la capacidad de sentir y emocionarse es la inspiración del ser humano para crear algo, se necesita de un ambiente de libertad de expresión, aprender a confiar en sí mismo e involucrarse cada vez más en su formación, el docente por su parte debe contribuir a formar en la sensibilidad y promover una relación dialógica entre la teoría, sensibilidady la práctica.

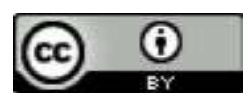

Este texto está protegido por una licencia Creative Commons 4.0.

Usted es libre para Compartir - copiar y redistribuir el material en cualquier medio o formato - y Adaptar eldocumento - remezclar, transformar y crear a partir del material- para cualquier propósito, incluso comercialmente, siempre que cumpla la condición de:

Atribución: Usted debe reconocer el crédito de una obra de manera adecuada, proporcionar un enlace a la licencia,e indicar si se han realizado cambios. Puede hacerlo en cualquier forma razonable, pero no de forma tal que sugieraque tiene el apoyo del licenciante o lo recibe por el uso que hace.

$\underline{\text { Resumen de licencia - Texto completo de la licencia }}$ 